\title{
Recent experimental activity on heavy-ion induced reactions within the NUMEN project
}

Francesco Cappuzzello ${ }^{1,2, *}$, Luis Acosta ${ }^{3}$, Clementina Agodi ${ }^{1}$, Carmen Altana ${ }^{1}$, Paulina Amador-Valenzuela ${ }^{4}$, Ismail Boztosun $^{5}$, Giuseppe A. Brischetto ${ }^{1,2}$, Salvatore Calabrese ${ }^{1,2}$, Daniela Calvo ${ }^{6}$, Vittoria Capirossi ${ }^{6,7}$, Diana Carbone ${ }^{1}$, Manuela Cavallaro $^{1}$, Efrain R. Chávez Lomeli ${ }^{3}$, Irene Ciraldo ${ }^{1,2}$, Mauro Cutuli ${ }^{1,2}$, Franck Delaunay ${ }^{1,2,8}$, Haris Djapo $^{9}$, Canel Eke ${ }^{5}$, Paolo Finocchiaro ${ }^{1}$, Suna Firat $^{5}$, Maria Fisichella ${ }^{1}$, Marcilei A. Guazzelli da Silveira ${ }^{10}$, Aylin Hacisalihoglu ${ }^{11}$, Felice Iazzi ${ }^{6,7}$, Gaetano Lanzalone ${ }^{1,12}$, Laura La Fauci ${ }^{1,2}$, Roberto Linares ${ }^{13}$, Nilberto H. Medina ${ }^{14}$, Mauricio Moralles ${ }^{15}$, José R. B. Oliveira $^{14}$, Athena Pakou ${ }^{16}$, Luciano Pandola ${ }^{1}$, Horia Petrascu ${ }^{17}$, Federico Pinna ${ }^{6,7}$, Giuseppe Russo ${ }^{1}$, Roberto B. B. Santos ${ }^{10}$, Onoufrios Sgouros ${ }^{1}$, Selçuk O. Solakci, ${ }^{5}$, George Souliotis ${ }^{18}$, Vasileios Soukeras ${ }^{1,2}$, Alessandro Spatafora ${ }^{1,2}$, Domenico Torresi $^{1}$, Salvatore Tudisco ${ }^{1}$, Aydin Yildirin ${ }^{5}$, Vinicius A. B. Zagatto ${ }^{13}$

for the NUMEN Collaboration

${ }^{1}$ Istituto Nazionale di Fisica Nucleare, Laboratori Nazionali del Sud, Catania, Italy

${ }^{2}$ Dipartimento di Fisica e Astronomia "Ettore Majorana", Università di Catania, Italy

${ }^{3}$ Instituto de Fisica, Universidad Nacional Autonoma de Mexico - Mexico City, Mexico

${ }^{4}$ Instituto Nacional de Investigaciones Nucleares - Ocoyoacac, Mexico

${ }^{5}$ Department of Physics, Akdeniz University - Antalya, Turkey

${ }^{6}$ Istituto Nazionale di Fisica Nucleare, Sezione di Torino, Italy

${ }^{7}$ DISAT, Politecnico di Torino, Italy

${ }^{8}$ LPC Caen, Normandie Universite, ENSICAEN, UNICAEN, CNRS/IN2P3 Caen, France

${ }^{9}$ Ankara University, Institute of Accelerator Technologies, Turkey

${ }^{10}$ Centro Universitario FEI - Sao Bernardo do Campo, Brazil

${ }^{11}$ Institute of Natural Sciences, Karadeniz Teknik University - Trabzon, Turkey

${ }^{12}$ Università degli Studi di Enna "Kore" - Enna, Italy

${ }^{13}$ Instituto de Fisica, Universidade Federal Fluminense - Niteroi, Brazil

${ }^{14}$ Instituto de Fisica, Universidade de Sao Paulo - Sao Paulo, Brazil

${ }^{15}$ Instituto de Pesquisas Energeticas e Nucleares IPEN/CNEN - Sao Paulo, Brazil

${ }^{16}$ Department of Physics and HINP, University of Ioannina - Ioannina, Greece

${ }^{17}$ IFIN-HH - Magurele, Romania

${ }^{18}$ Department of Chemistry, National and Kapodistrian University of Athens, Athens, Greece

\begin{abstract}
The possibility to use a special class of heavy-ion induced direct reactions, such as double charge exchange reactions, is discussed in view of their application to extract information that may be helpful to determinate the nuclear matrix elements entering in the expression of neutrinoless double beta decay half-life. The strategies adopted in the experimental campaigns performed at INFN - Laboratori Nazionali del Sud are briefly described, emphasizing the advantages of the multi-channel approach to nuclear reaction data analysis.
\end{abstract}

\section{Introduction}

Charge exchange $(\mathrm{CE})$ reactions are nuclear processes in which the individual balance of neutrons and protons inside the projectile and the target is modified, while keeping the overall number of nucleons unchanged. In the isospin formalism the $\mathrm{CE}$ promote isovector excitations in both the projectile and target. $\mathrm{CE}$ are thus selective tools to emphasize the role of isospin in both nuclear structure and reaction studies. Relevant aspects of single charge exchange (SCE) induced by light projectile have been discussed in several articles, among which excellent reviews are in ref. [1] [2] [3]. For heavy-ion induced CE we refer to the recent review [4]. An especially appealing aspect is the possibility to use $\mathrm{CE}$ to investigate the spinisospin-dependent response of nuclei.

Recently, particular interest has been given to double charge exchange (DCE) processes [5] [6] [7] as they can give access to the nuclear response to second order isospin operators, which also promote double beta decays.

SCE and DCE studies have a genuine nuclear physics interest, since they reflect a key aspect of nuclear dynamics. However, beyond that, there is an even broader impact of such investigations because of their relevance for nuclear beta decay, thus connecting strong and weak interactions.

In this context, the NUMEN and NURE projects at INFNLNS laboratory in Catania [8] [9] aim at exploring heavyion induced single and double charge exchange (DCE)

\footnotetext{
* Corresponding author: cappuzzello@lns.infn.it
} 
reactions in a systematic approach. The main goal is to extract information on nuclear matrix elements for SCE and DCE processes and to identify the possible connections with double beta decay.

Detailed information about these projects are found in recent articles, which we refer to [10] [11] [12] for both scientific and technological aspects.

In the present paper, the global strategy and methodologies of the above mentioned programmes, the challenges of the experimental activities together with the status of the already performed experimental runs are briefly outlined.

\section{The motivation}

Neutrinoless double beta decay $(0 v \beta \beta)$ is an hypothetical process in which a "parent" nucleus is spontaneously transformed into a "daughter" isobar by the conversion of two neutron/protons into two protons/neutrons. The process is accompanied by the emission of two electrons (or positrons), which guarantee also electric charge conservation. If detected, $0 v \beta \beta$ would provide evidence for the Majorana nature of the neutrino (i.e. the equivalence of neutrino and antineutrino) and give access to the neutrino mass [13] [14] [15] [16]. Under the hypothesis that the neutrino, electrons and nuclear degrees of freedom can be separated in $0 v \beta \beta$, the decay rate explicitly depends on the nuclear transition probability, accounted for in the so-called Nuclear Matrix Element (NME). The 0v $\beta \beta$ NMEs are very sensitive to nuclear physics involved in decay, since they probe the nuclear wave functions of the initial and final states. In particular, the nuclear response to high-momentum second-order isospin operators is probed in $0 v \beta \beta$, similarly to what happen in DCE reactions [17]. However, there are no experimental methods to directly measure $0 v \beta \beta$ NMEs and accurate theoretical calculations are challenging, leading to discrepancy factors larger than two, as presently reported in literature [13]. Thus experimental inputs from SCE and DCE studies are useful to help evaluate the $0 v \beta \beta$ NMEs and to constrain the calculations. In this context, an experimental campaign has started at the INFN-Laboratori Nazionali del Sud in Catania using the MAGNEX large acceptance magnetic spectrometer [18]. It focuses on the study of heavy-ion induced DCE reactions and competing reaction channels involving the nuclei of interest for $0 v \beta \beta$ decay.

The possible connections of DCE measured crosssections with double beta decay NMEs and, consequently, with the still unsolved neutrino puzzles are among the goals of the present studies.

\section{Heavy-ion induced double charge exchange reactions}

Challenging experimental issues must be addressed to measure heavy-ion induced DCE cross sections. Indeed one needs to detect heavy ions with good isotopic separation and energy resolution in a wide angular range, including zero-degree, in order to distinguish transitions to individual states and explore a wide momentum transfer range. In addition, the rather tiny DCE cross sections (down to few $\mathrm{nb}$ ) demand for high experimental sensitivity, which strongly depends on the rejection capability against unwanted spurious events. These latter may be generated by competing reaction processes, some of which are more likely to occur than DCE. For this reason highly selective particle identification is a necessary prerequisite for these experiments. In addition, spurious data associated to a wrong determination of detection parameters could casually be misinterpreted as valid DCE data. Characterizing the relevant background sources can be subtle and require stringent conditions for the experimental set-up as well as a careful study of the detection response [19].

Recently, accurate cross sections for heavy-ion DCE reactions have been measured at the INFN-LNS laboratory, thanks to the use of the MAGNEX spectrometer.

The accurate description of heavy-ion induced DCE is a demanding task also for nuclear theory. From the reaction theory side initial and final state interactions of projectile and target systems need to be under control and the reaction amplitude must be described properly. In this context direct reaction theory can provide the suitable toolbox. Nuclear structure theory is asked to provide accurate input for modelling both projectile and target [20].

Recently, new theoretical developments for the reaction mechanism involved in heavy ion induced reactions have been achieved alongside. Presently this activity is in progress within the NUMEN project [21] [22] [23] [24].

\section{The experiments}

The NUMEN experiments are performed at INFN-LNS using the K800 Superconducting Cyclotron to accelerate beams and the MAGNEX large acceptance magnetic spectrometer for the detection of the ejectiles.

MAGNEX is a large acceptance magnetic device consisting of a large aperture vertically focusing quadrupole and a horizontally bending dipole magnet. A hybrid Focal Plane detector (FPD) featuring a gaseous tracker and a wall of silicon pad sensors is mounted at the focal plane to detect the reaction ejectiles [25] [26].

MAGNEX was designed to investigate processes characterized by very low yields, guaranteeing the identification of heavy ions with quite high mass $(\Delta \mathrm{A} / \mathrm{A} \sim 1 / 160)$, angle $\left(\Delta \theta \sim 0.2^{\circ}\right)$ and energy resolutions $(\Delta \mathrm{E} / \mathrm{E} \sim 1 / 1000)$, within a large solid angle $(\Omega \sim 50 \mathrm{msr})$ and momentum range $(-14 \%<\Delta \mathrm{p} / \mathrm{p}<+10 \%)$. Highresolution measurements for quasi-elastic processes, characterized by differential cross-sections as low as tens of nb/sr, were already performed with this setup [27] [28]. A crucial feature is the implementation of a technique of trajectory reconstruction, based on differential algebraic techniques and the accurate mapping of the spectrometer magnetic fields [29] [30] [31] [32], which solves the equation of motion of each detected particle to $10^{\text {th }}$ order [33]. This is a unique characteristic of MAGNEX, which guarantees the above mentioned performances and its relevance in the worldwide scenario of heavy-ion physics. 
The experimental activity with accelerated beams proposed and presently in progress consists of two main classes of experiments, aiming at the exploration of the two directions of isospin lowering $\tau^{-} \tau^{-}$and rising $\tau^{+} \tau^{+}$, characteristic of $\beta^{-} \beta^{-}$and $\beta^{+} \beta^{+}$decays, respectively.

In particular, the $\beta^{+} \beta^{+}$direction in the target excitation modes is investigated using a ${ }^{18} \mathrm{O}^{8+}$ beam and measuring the $\left({ }^{18} \mathrm{O},{ }^{18} \mathrm{Ne}\right)$ DCE transitions, together with other reaction channels involving same beam and target. Similarly, the $\beta^{-} \beta^{-}$direction is explored via the $\left({ }^{20} \mathrm{Ne},{ }^{20} \mathrm{O}\right)$ reaction, using a ${ }^{20} \mathrm{Ne}^{10+}$ beam and detecting the reaction products from the DCE channel and from the other open reaction channels.

In each case, the experiments are organized in order to explore together with the DCE reactions of interest, all the relevant quasi-elastic processes occurring in the projectile-target collision. In this way, we can get supplementary information from the detailed study of the reaction network, profiting of the strong selectivity of quasi-elastic processes. The nucleus-nucleus interaction can be extracted from elastic scattering data and applied to the evaluation of the distorted waves in each direct reaction channel. Inelastic scattering data give selective access to the role of the deformation in the coupled channel reaction network equations. Single nucleon transfer reactions shed light on the role of single particle configurations in the involved nuclear many body states. Two nucleon transfer reactions probe pairing correlations, while SCE give access to 1particle-1hole excitations. All of these information are provided in a consistent experimental framework and analysed in a multi-channel approach, based on quantum scattering theory of coupled channels. A unique opportunity is thus searched by NUMEN to build an over-constrained set of nuclear structure information, promoting the development of a new approach to $0 v \beta \beta$ NMEs calculations.

Investigations of the two classes of experiments have been started in the recent years with the present facility. These studies highlighted the strengths and the limiting aspects of the adopted technique, providing first valuable information on relevant nuclear structure aspects for a number of double beta decay emitter candidates [8]. This activity has also given precious information to guide the major upgrade of the facility toward much higher beam intensity, which is presently going on.

\subsection{Experiments with ${ }^{18} \mathrm{O}$ beam}

For the experiments of this class, the reaction channels of our interest are listed below:

- $\left({ }^{18} \mathrm{O},{ }^{18} \mathrm{O}\right)$ elastic and inelastic scattering

- $\left({ }^{18} \mathrm{O},{ }^{18} \mathrm{Ne}\right) \mathrm{DCE}$ reaction

- $\left({ }^{18} \mathrm{O},{ }^{18} \mathrm{~F}\right) \mathrm{SCE}$ reaction

- $\left({ }^{18} \mathrm{O},{ }^{20} \mathrm{Ne}\right)$ two-proton pickup reaction

- $\left({ }^{18} \mathrm{O},{ }^{19} \mathrm{~F}\right)$ one-proton pickup reaction

- $\left({ }^{18} \mathrm{O},{ }^{16} \mathrm{O}\right)$ two-neutron stripping reaction

- $\left({ }^{18} \mathrm{O},{ }^{17} \mathrm{O}\right)$ one-neutron stripping reaction

One of the main challenges of such experiments is the measurement at very forward angles, including zerodegree. This is performed by placing the spectrometer with its optical axis at $+3^{\circ}$ with respect to the beam axis. Thanks to its large angular acceptance, the range $-2^{\circ}<$ $\theta_{\text {lab }}<9^{\circ}$ is explored. The MAGNEX quadrupole and dipole magnetic fields are set in order that the incident beam, after passing through the magnets, reaches a region besides the FPD. For this class of experiments, in fact, the incident ion beam $\left({ }^{18} \mathrm{O}^{8+}\right)$ has higher magnetic rigidity $(\mathrm{B} \rho)$ than the ejectiles of interest (namely fully stripped ${ }^{18} \mathrm{Ne},{ }^{18} \mathrm{~F},{ }^{20} \mathrm{Ne},{ }^{19} \mathrm{~F},{ }^{16} \mathrm{O},{ }^{17} \mathrm{O}$ ions). The beam stops in a Faraday cup, placed in the high-B $\rho$ region besides the FPD, which measures the collected charge for each run.

${ }^{116} \mathrm{Sn},{ }^{76} \mathrm{Se}$ and ${ }^{48} \mathrm{Ti}$ are the targets of interest for $0 v \beta \beta$, which have already been explored by NUMEN, via $\left({ }^{18} \mathrm{O},{ }^{18} \mathrm{Ne}\right)$ reaction at 15 and $22 \mathrm{AMeV}$. The purpose was to study the ${ }^{116} \mathrm{Sn} \rightarrow{ }^{116} \mathrm{Cd},{ }^{76} \mathrm{Se} \rightarrow{ }^{76} \mathrm{Ge}$ and ${ }^{48} \mathrm{Ti} \rightarrow{ }^{48} \mathrm{Ca}$ transitions and the competing channels as shown in Fig. 2. A dedicated run was also performed on ${ }^{12} \mathrm{C}$ target, in order to compare reaction models with an easier light system. The reduction and analysis of the collected data from these campaigns is presently in progress. First results have been already published in ref. [34] [35] [36] [37].

3.2. Experiments with ${ }^{20} \mathrm{Ne}$ beam $\left(\beta^{-} \beta^{-}\right.$direction) In the class of experiments triggered by ${ }^{20} \mathrm{Ne}^{10+}$ beams, the reaction channels we are interested are the following:

- $\left({ }^{20} \mathrm{Ne},{ }^{20} \mathrm{Ne}\right)$ elastic and inelastic scattering

- $\left({ }^{20} \mathrm{Ne},{ }^{20} \mathrm{O}\right)$ DCE reaction

- $\left({ }^{20} \mathrm{Ne},{ }^{20} \mathrm{~F}\right) \mathrm{SCE}$ reaction

- $\left({ }^{20} \mathrm{Ne},{ }^{18} \mathrm{O}\right)$ two-proton stripping reaction

- $\left({ }^{20} \mathrm{Ne},{ }^{19} \mathrm{~F}\right)$ one-proton stripping reaction

- $\left({ }^{20} \mathrm{Ne},{ }^{22} \mathrm{Ne}\right)$ two-neutron pickup reaction

- $\left({ }^{20} \mathrm{Ne},{ }^{21} \mathrm{Ne}\right)$ one-neutron pickup reaction.

For these experiments, the incident beam $\left({ }^{20} \mathrm{Ne}^{10+}\right)$ has a lower magnetic rigidity compared to to the ejectiles of interest. Thus, for a fixed magnetic field setting, the beam is more bent than the ejectiles of interest. The spectrometer optical axis is typically placed at $-3^{\circ}$, thus the covered angular range is $-8^{\circ}<\theta_{\text {lab }}<+3^{\circ}$. The quadrupole and dipole magnetic fields are set in order that the ${ }^{20} \mathrm{Ne}^{10+}$ beam reaches the low-B $\rho$ region besides the FPD. The ${ }^{20} \mathrm{Ne}^{9+}$ and ${ }^{20} \mathrm{Ne}^{8+}$ components of the beam emerging for the target could be a source of background in these experiments. They are significantly reduced by the use of a supplementary stripper foil mounted after the target itself [38].

The systems already experimentally explored using the $\left({ }^{20} \mathrm{Ne},{ }^{20} \mathrm{O}\right)$ reaction at $15 \mathrm{AMeV}$ are the ${ }^{116} \mathrm{Cd}$ target (to study the ${ }^{116} \mathrm{Cd} \rightarrow{ }^{116} \mathrm{Sn}$ transition), the ${ }^{130} \mathrm{Te}$ (for the ${ }^{130} \mathrm{Te}$ $\rightarrow{ }^{130} \mathrm{Xe}$ ) and the ${ }^{76} \mathrm{Ge}$ (for the ${ }^{76} \mathrm{Ge} \rightarrow{ }^{76} \mathrm{Se}$ ). The data reduction and analysis is in progress and the first results from some reaction channels have been recently published [39] [40] [41] [42].

A scheme of the transitions already studied in the experimental runs is shown in Fig. 2. 

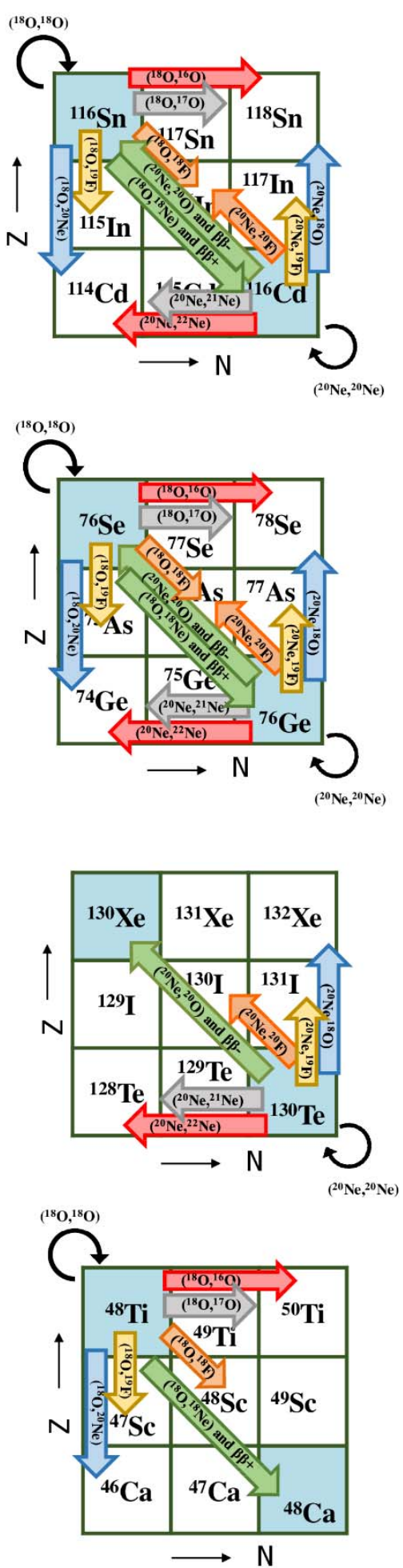

Fig. 2 Scheme of the reaction channels already studied within the NURE and NUMEN experiments.

\section{Conclusions}

A systematic study of heavy-ion induced DCE reactions and of the other reaction channels characterized by the same projectile and target is in progress at INFN-LNS. The main goal is to investigate the nuclear response to DCE reactions for all the isotopes candidate for $0 v \beta \beta$ decay towards the determination of $0 v \beta \beta$ NMEs.

\section{Acknowledgments}

This project has received funding from the European Research Council (ERC) under the European Union's Horizon 2020 research and innovation programme (grant agreement No 714625).

\section{References}

[1] Alford, W.P.; Spicer, B.M. Nucleon charge-exchange reactions at intermediate energy. Adv. Nucl. Phys. 1998, $1,24$.

[2] Osterfeld, F. Nuclear spin and isospin excitations. Rev. Mod. Phys. 1992, 64, 491..

[3] Taddeucci, T.N.; et al. The $(p, n)$ reaction as a probe of beta decay strength. Nucl. Phys. A 1987, 469, 125. .

[4] Lenske, H.; Cappuzzello, F.; Cavallaro, M.; Colonna, M. Heavy ion charge exchange reactions as probes for nuclear $\beta$-decay. Prog. Part. Nucl. Phys. 2019, 109, 103716.

[5] F. Cappuzzello et al., Eur. Phys. J. A 51, 145 (2015).

[6] Cappuzzello, F.; Cavallaro, M.; Agodi, C.; Bondì, M.; Carbone, D.; Cunsolo, A.; Foti, A. Heavy-ion double charge exchange reactions: A tool toward $0 v \beta \beta$ nuclear matrix elements. Eur. Phys. J. A 2015, 51, 145.

[7] Cappuzzello, F., and Agodi, C. (2021). The NUMEN Project: Shedding Light on Neutrinoless Double Beta Decay by Heavy-Ion Nuclear Reactions. Nucl, Phys. News, in press, DOI 10.1080/10619127.2021.1881368.

[8] Cappuzzello, F., Agodi, C., Cavallaro, M., Carbone, D., Tudisco, S., Lo Presti, D., et al.. (2018). The NUMEN project: NUclear Matrix Elements. Eur. Phys. J. A. 54, 72.

[9] Cavallaro, M., Aciksoz, E., Acosta, L., Agodi, C., Auerbach, N., Bellone, J. I., et al., (2017). "NURE: an ERC project to study nuclear reactions," in Proceedings, 55th International Winter Meeting on Nuclear Physics Bormio, (Bormio). doi: 10.22323/1.302.

[10] P. Finocchiaro et al., Universe 2020, 6, 129; doi:10.3390/universe6090129.

[11] C. Agodi et al., Universe 2021, 7, 72. https://doi.org/10.3390/universe7030072.

[12] F. Cappuzzello et al., The NUMEN Project: An Update of the Experimental Campaigns. Front. Astron. Space Sci. 8:668587. doi: 10.3389/fspas.2021.668587.

[13] H. Ejiri, J. Suhonen, K. Zuber, Physics Reports 797 (2019) 1.

[14] J. Engel, J. Menéndez, Rep. Progr. Phys. 60 (2017) 046301.

[15] S. Dell'Oro, S. Marcocci, and F. Vissani, Phys. Rev. D 90, 033005 (2014).

[16] J. Vergados, H. Ejiri, F. Šimkovic, Rep. Progr. Phys. 75 (2012) 106301.

[17] Cappuzzello, F., and Cavallaro, M. (2020). Nuclear Response to Second-Order Isospin Probes in Connection to Double Beta Decay. Universe. 6, 217.

[18] F. Cappuzzello et al. The MAGNEX spectrometer: Results and perspectives. Eur. Phys. J. A (2015) 51:145.

[19] S. Calabrese et al. Analysis of the background on cross section measurements with the MAGNEX spectrometer: The (20Ne,20O) Double Charge Exchange case. Nuclear Inst. and Methods in Physics Research, A 980 (2020) 164500 . 
[20] N. Shimizu, J. Menendez, and K. Yako, Phys. Rev. Lett. 120,142502 (2018).

[21] H. Lenske, J.I. Bellone, M. Colonna, J. A. Lay, Phys. Rev. C 98 (4), 044620 (2018)..

[22] E. Santopinto, H. García-Tecocoatzi, RIM Vsevolodovna, J. Ferretti, Phys. Rev. C 98 (6), 061601 (2018).

[23] J.I. Bellone, S. Burrello, M. Colonna, J. A. Lay, H. Lenske, Phys. Lett. B 807, 135528 (2020).

[24] Lenske, H.; Bellone, J.; Colonna, M.; Gambacurta, D. Nuclear Matrix Elements for Heavy Ion Sequential Double Charge Exchange Reactions. Universe 2021, 7, 98. https://doi.org/10.3390/universe7040098.

[25] Torresi, D., Sgouros, O., Soukeras, V., Cavallaro, M., Cappuzzello, F., Carbone, D., et al. (2021). An upgraded focal plane detector for the MAGNEX spectrometer. Nucl. Instr. and Meth. A. 989, 164918.

[26] M. Cavallaro et al. The low-pressure focal plane detector of the MAGNEX. Eur. Phys. J. A (2012) 48: 59. DOI 10.1140/epja/i2012-12059-8.

[27] J.R.B. Oliveira et al., Journal of Physics G: Nuclear and Particle Physics, 40 (2013) 105101.

[28] D. Pereira et al. Phys. Lett. B 710 (2012) 426.

[29] A. Lazzaro et al., Nucl. Instr. and Methods A 591, 394 (2008)..

[30] A. Lazzaro et al., Nucl. Instr. and Methods A 585, 136 (2008).

[31] A. Lazzaro et al., Nucl. Instr. and Methods A 570, 192 (2007).

[32] A. Lazzaro et al., Nucl. Instr. and Methods A 602, 494 (2009).

[33] F. Cappuzzello et al., Eur. Phys. J. A 52 (2016) 169.

[34] O. Sgouros et al., Phys. Rev. C, accepted.

[35] D. Carbone et al., Universe 7 (2021) 58.

[36] J. L. Ferreira et al., Phys. Rev. C 103 (2021) 054604.

[37] M. Cavallaro et al., Frontiers in Astron. and Space Science 8 (2021) 659815.

[38] M. Cavallaro et al., Results in Phys. 13 (2019) 102191.

[39] A. Spatafora et al., Phys. Rev. C 100 (2019) 034620.

[40] V. Soukeras et al., Results in Physics, (2021) submitted.

[41] S. Calabrese et al., Nucl. Instr. and Meth. A, 980 (2020) 164500 .

[42] S. Calabrese et al., Acta Phys. Pol. B 49 (2018) 275. 